\title{
A Novel Design of a Robust Ten-Port Microwave Reflectometer With Autonomous Calibration by Using Neural Networks
}

\author{
Juan Monzó-Cabrera, Juan L. Pedreño-Molina, Antonio Lozano-Guerrero, and Ana Toledo-Moreo
}

\begin{abstract}
In this study, a novel ten-port waveguide microwave sensor is designed, implemented, calibrated and tested in order to obtain the reflection coefficient magnitude and phase. This reflectometer is based on the well known six-port structure but the number of detectors has been increased to eight in order to improve the sampling procedure of the standing wave present within the waveguide. In addition, a learning method based on neural networks' usage has been implemented for autonomous calibration from the data collected by a vector network analyzer. An automated procedure consisting of a moving sample within a multimode cavity has enabled different reflection coefficients to be obtained. Neural networks have been employed in order to learn the relationship between the actual reflection parameter and the acquired signals from eight power detectors. This novel device has been calibrated with a neural architecture based on radial basis functions and the error of device measurements has been analyzed. This new design and the incorporated neural network calibration allow one to avoid problems caused by fault or nonlinearity of the detectors, and to get robustness, flexibility and adaptability characteristics for the presented device.
\end{abstract}

Index Terms-Autonomous calibration, neural networks, scattering parameters, six-port, ten-port microwave reflectometer.

\section{INTRODUCTION}

D IFFERENT alternatives have been proposed to estimate the scattering parameters in microwave-heating processes. Traditional methods for these techniques [1] are based, for instance, on slotted lines or impedance bridges [2] or circuit designs based on directional couplers [3]. Another possibility is the so-called six-port reflectometer [4], [5], as well as the employment of conventional network analyzers. However, in the case of the six-port, several advantages are found versus network analyzers such as portability, lower price and simple structure. Additionally, for high-power signals, power measurement devices of six-port are much easier to design than the circuits which are used in traditional network analyzers.

The six-port methodology, designed by Hoer in 1972, is an inexpensive solution that avoids the use of network analyzers. It is based on the employment of simple power detectors (like

Manuscript received April 15, 2008; revised July 30, 2008. First published November 18, 2008; current version published December 05, 2008.

J. Monzó-Cabrera, J. L. Pedreño-Molina, and A. Lozano-Guerrero are with the Departamento de Tecnologías de la Información y las Comunicaciones, Universidad Politécnica de Cartagena, 30202 Cartagena, Murcia, Spain (e-mail: Juan.Monzo@upct.es; Juan.PMolina@upct.es; Antonio.Lozano@upct.es).

A. Toledo-Moreo is with the Departamento de Tecnología Electrónica, Universidad Politécnica de Cartagena, 30202 Cartagena, Murcia, Spain (e-mail: Ana.Toledo@upct.es).

Digital Object Identifier 10.1109/TMTT.2008.2007318

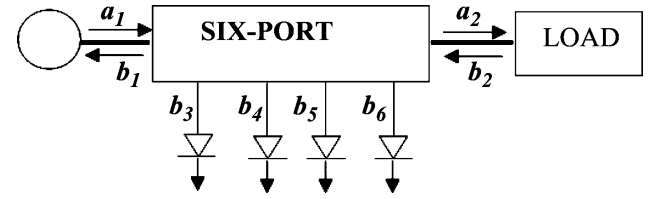

Fig. 1. Six-port reflectometer structure.

diodes or thermistors). Due to noise presence and the nonlinear characteristics of the power detectors, a calibration procedure has to be carried out for the six-port within the working bandwidth to ensure the accuracy of the measurements.

\section{A. Operation Principle of a Six-Port Reflectometer}

A waveguide six-port reflectometer consists of a simple waveguide with six ports: one of them connected to the microwave source, the other one to the unknown load and four coaxial ports loaded with power detectors. The coaxial ports extract samples of the waveguide incident and reflected waves by using the central conductor of the coaxial ports as an antenna or probe. From the outputs of the detectors, a numerical relationship can be theoretically obtained to determine the reflection coefficient [2]. This numerical expression combines the value of nine parameters, which are very sensitive to noise effects in the measurements, when trying to determine the desired reflection coefficient value defined as $\Gamma=b_{2} / a_{2}$, as shown in Fig. 1.

The six-port calibration procedure is based on finding the numerical solution for the system of equations shown, as follows in (1), with $M_{i}$ and $N_{j}$ being complex constants; therefore, eight equations must be evaluated in order to infer $M_{i}$ and $N_{j}$ values:

$$
\begin{aligned}
& b_{3}=M_{3} \cdot a_{2}+N_{3} \cdot b_{2} \\
& b_{4}=M_{4} \cdot a_{2}+N_{4} \cdot b_{2} \\
& b_{5}=M_{5} \cdot a_{2}+N_{5} \cdot b_{2} \\
& b_{6}=M_{6} \cdot a_{2}+N_{6} \cdot b_{2} .
\end{aligned}
$$

The most extended techniques for solving this system are based on terminating the six-port with at least four standard loads [2], sliding terminations [6] or the thru-reflect-line (TRL) method [7]. Another possibility is described in [8] where five loads with the same module are used for the calibration, eliminating ill-conditioned configurations.

The numerical solution can be graphically represented in the complex impedances plane by means of three circles, whose intersections provide the desired solution for $\Gamma$, and techniques 
to decrease the number of parameters in (1) can be found in [6] by exploiting circular symmetries with respect to the complex axes.

\section{B. Six-Port Calibration Techniques}

The main problems of the previous calibration methodology are due to error in the measurements such as noise and nonlinearity effects. As a consequence, the intersection point in the complex plane is extended to a wider region in which several solutions are possible. In this way, efforts have been made to numerically find the solution in presence of noise such as methods based on least squares algorithms for mean power error Gaussian distributions [9] or techniques based on Fourier analysis of the six-port parameters [10].

However, in all these methods the nonlinearity of the diodes and the presence of noise are not considered. Additionally, uncertainty of connectors, transmission lines or diodes, may increment the final error in measurements, which is more relevant when high power levels are considered. Other techniques for the calibration of diodes' effects have been proposed, such as linear approximation around the working frequency [8], [11], inclusion of a correction factor depending on temperature [12] or sliding terminations for the calibration of both the diode and the six-port [13].

Finally, methods based on artificial neural networks are used in [12] or [14] for both the six-port and diode calibration by using electromagnetic simulation platforms. Neural networks in those works avoid numerical modeling of the physical behavior of all the components at the six-port device.

From those works, measurement based on neural network learning procedures seems to be the best solution for the calibration of a reflectometer device because they are able to establish a precise relationship between the outputs of diodes and the $S_{11}$ parameter by means of an adaptive process. This learning procedure is able to counteract effects such as noise or nonlinearity of the diodes. Additionally in [15], both $\left|S_{11}\right|$ training and validation data are extracted by a simple procedure of irradiating a sample of any material at different distances from the microwave source.

In this study, a novel ten-port microwave sensor for both $S_{11}$ magnitude and phase measurement, which is calibrated with neural networks, has been developed in order to improve the sixport performance. Six-port internal characteristics have been modified and a neural process has been implemented for calibration and $S_{11}$ measurement.

Thus, a ten-port structure incorporating neural-network calibration has been designed and subsequently manufactured. Aspects such as accuracy, adaptability and robustness in the measurement procedure have been tested by using a microwave heating multimode cavity as impedance generator. All the tests have validated the proposed mechanical design, electronic assembly and the neural calibration and measurement methods for low microwave power levels.

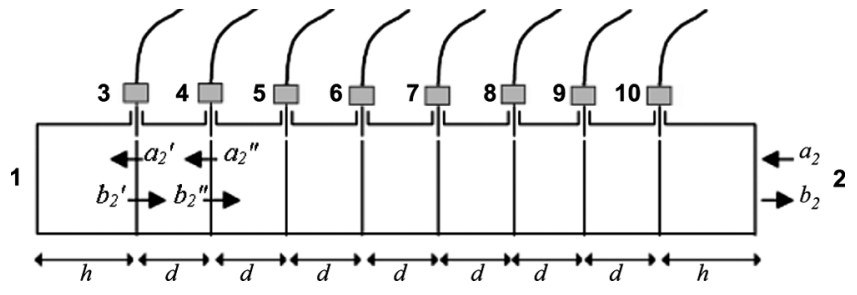

Fig. 2. Scheme of the ten-port structure with port numeration.

\section{MethodS AND EXPERIMENTAL SETUP}

\section{A. Ten-Port Reflectometer Implementation}

In this study, the microwave reflectometer (Fig. 2) is formed by a WR-340 rectangular waveguide with ten ports, two of them corresponding to the input and output ports of the waveguide. Eight coaxial ports have been placed at the centre of the biggest face of the waveguide, in order to sample the standing wave of the $\mathrm{TE}_{10}$ mode at its maximum value.

The central conductor of the SMA coaxial connectors penetrates inside the rectangular cross section with a length of $16 \mathrm{~mm}$, which provides a power coupling of $-23 \mathrm{~dB}$. This value has been chosen in order to ensure that the power detectors are never saturated since the output power of the network analyzer, which was used in this study as the microwave source and reference instrument, was set to $27 \mathrm{dBm}$ and the saturation power level of the power detectors is $10 \mathrm{dBm}$. The distance between each coaxial port is selected as a function of the $\mathrm{TE}_{10}$ wavelength, resulting in $d=22 \mathrm{~mm}$, with separations of $35 \mathrm{~mm}$ from the extremes. The final length of the ten-port is $224 \mathrm{~mm}$.

The selection of eight sampling ports instead of the four probes used in the six-port has been considered to improve the accuracy of the final result since a neural network is used for the ten-port calibration. In fact, if the network is designed appropriately, the prediction process is more precise when much information about the same function is acquired.

Moreover, this redundancy and the proposed neural-network method for the calibration permits the modification of the behavior of the ten-port sensor to scenarios in which the detection process in whatever port is damaged. In contrast, the six-port structure employs the minimal number of samples necessary to solve the numerical equation, which impedes adaptability to changes in the system such as detector failure.

As previously commented, the ten-port device presented in this paper has been designed to estimate the complex value of $S_{11}$ at $2.45 \mathrm{GHz}$, when low microwave power is supplied from the microwave source, a ZVRE Rohde \& Schwarz vector network analyzer (VNA) in this case. The WR-340 rectangular waveguide has been manufactured with aluminum of $8 \mathrm{~mm}$ thickness. The eight SMA coaxial connectors have been loaded with microwave integrated circuits containing the power detectors, and one data acquisition device has been employed to multiplex the outputs of the power-meter boards and convert them to a digital format. Both the calibration and the measurement procedures are computed by means of neural networks 


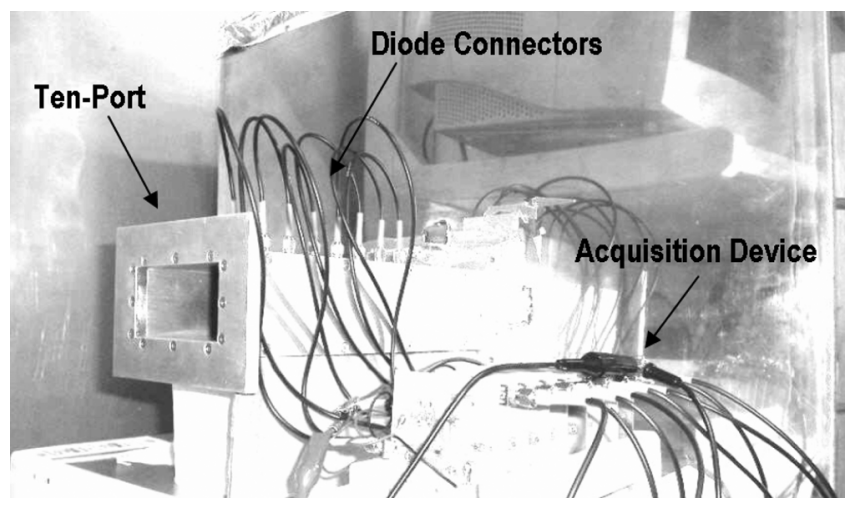

Fig. 3. Experimental scenario for microwave ten-port operation.

in a personal computer. Fig. 3 shows the experimental setup employed in all tests.

As it can be observed in Fig. 3, the load connected to the ten-port reflectometer consists of a microwave $60 \mathrm{~cm} \times 60 \mathrm{~cm} \times$ $60 \mathrm{~cm}$ multimode cavity. Within this cavity, a nylon computercontrolled cartload moves a dielectric sample to different positions in order to achieve different reflection coefficients for the learning mode or calibration stage.

The ZVRE VNA is connected to the computer in order to acquire the reference measurements for $S_{11}$ in each sample position. In this way, the VNA acts both as the microwave source and calibrated sensor during the calibration stage. In this case the operating frequency for all the measurements was set to $2.45 \mathrm{GHz}$ and the power delivered by the VNA was $0.5 \mathrm{~W}$. The VNA was calibrated by using a WCK-340-HP waveguide calibration kit from Continental Microwave. This calibration kit uses short, matched loads and through connection as reference measurements.

It must be remarked that the $S_{11}$ values measured by the VNA were only used to train the neural networks during the ten-port calibration stage. After that stage the ten-port was able to predict $S_{11}$ without the need of any VNA. Although other approaches were possible, such as the use of theoretically known impedances, this one was chosen since it allowed computer control and high precision reference measurements.

All the detectors employed at the coaxial ports in this experimental setup were LTC5530 nonlinear power detectors, whose input working range is from -30 to $10 \mathrm{dBm}$ and its operating bandwidth covers from $300 \mathrm{MHz}$ to $7 \mathrm{GHz}$. The typical output voltage range covers from $100 \mathrm{mV}$ to $3.6 \mathrm{~V}$. Since the curve for the output voltage versus the input power was not provided by the manufacturer at $2.45 \mathrm{GHz}$, this relationship was experimentally determined by using the VNA as an RF signal generator and a conventional multimeter for measuring the output voltage. Fig. 4 shows the detector output voltage versus the input power at $2.45 \mathrm{GHz}$ and $25^{\circ} \mathrm{C}$.

Finally, the working methodology of this experimental setup is as follows. The VNA irradiates electromagnetic energy that is propagated across the ten-port reflectometer. This energy reaches a loaded multimode oven so that a part of the microwave power is absorbed by the dielectric sample and the rest is reflected towards the analyzer that measures the reference complex value of the reflection coefficient. Thus, inside the

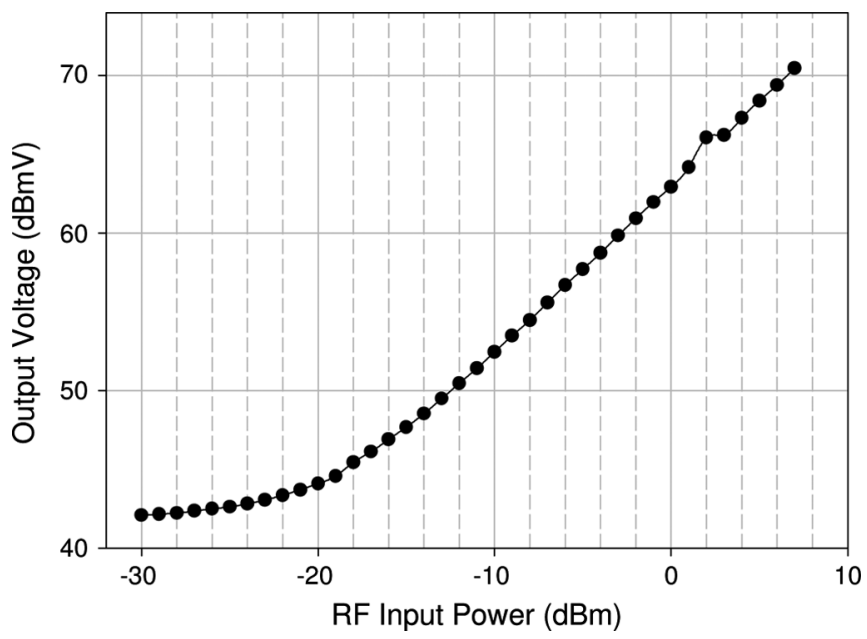

Fig. 4. Output voltage versus input power for LTC5530 nonlinear power detectors at $2.45 \mathrm{GHz}$.

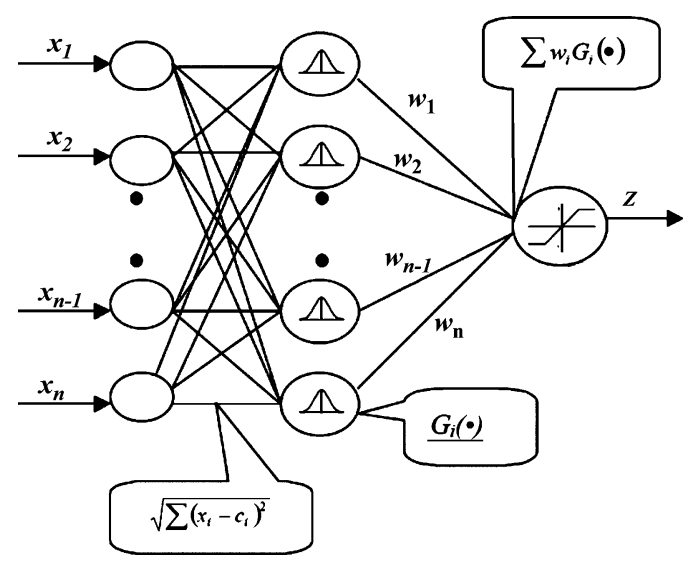

Fig. 5. Scheme of the neural network for $S_{11}$ magnitude and phase prediction, where $x_{i}, G_{i}$ and $z$ represent the levels for the input, hidden and output, respectively.

ten-port waveguide section a standing wave is generated. Eight coaxial probes sample this standing wave and the microwave power detectors measure the power at each of these eight locations. Each input power at the detectors is converted to an output voltage signal and this voltage is captured by a data acquisition board. Both the VNA reference measurement and the eight output voltages are supplied to the computer in order to train the neural network, which is dedicated to find the relationship of the VNA-measured $S_{11}$ parameter and the eight voltages of the power detectors for different positions of the sample inside the oven.

\section{B. Neural Network Training Methodology}

The well-known radial basis function neural network [16] has been selected to estimate both the magnitude and phase of $S_{11}$ from the eight voltage values provided by the power detectors. Fig. 5 shows the neural architecture used in this study.

This neural architecture consists of a nonlinear hidden level and other linear output level in which the contribution of the signals pondered by the $G_{i}$ Gaussians activation functions of the neurons, are combined to provide the $S_{11}$ parameter at the 


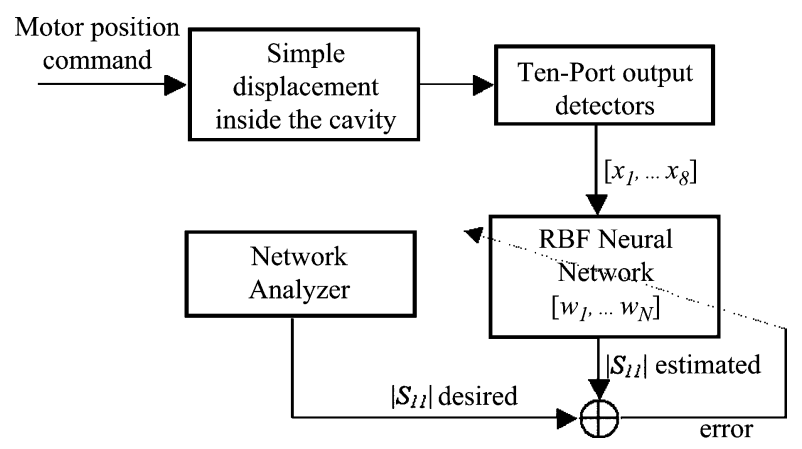

Fig. 6. Calibration procedure scheme of the ten-port by using the radial basis function $(\mathrm{RBF})$ neural network.

working frequency. These functions are defined by the $c_{i}$ adaptive centroids and a constant variance value for all the Gaussians expressions.

The $c_{i}$ centroids determine the segmentation of the input space of the $x$ input vector. The components $x_{i}$ of the $x$ vector form the input level of the neural network, while $G_{i}$ form the hidden level. The output of the network is obtained by means of the vectorial expression $\boldsymbol{W} \times \boldsymbol{G}$ in the output linear level. In this case, the input vector is formed by the eight voltage values provided by the LTC5530 detectors, whereas the output of the network estimates $\left|S_{11}\right|$. A similar scheme is used for the estimation of $S_{11}$ phase.

As it is shown in Fig. 5, these inputs are projected over the hidden level by means of the evaluated Euclidean distance. In this level, the radial basis functions, given by $G_{i}$, evaluate the contribution of each $x$-component with respect to the $c_{i}$ centroids. Finally the outputs of each Gaussian function are pondered by the $w_{i}$ neural weights, and projected toward the output linear level in which all the contributions are summed to the estimation for the $S_{11}$ parameter.

In order to design a calibration procedure for this ten-port, it is necessary to acquire as many values as possible for $\left|S_{11}\right|$ and its correspondent eight-length voltage output vector from the power detector circuits. To do this, a system of variable loads must be implemented to ensure that values for $S_{11}$ cover all the multimode oven range of impedances. This is a requirement for the correct training procedure of the neural network weights $\left(w_{i}\right)$. In this study, the proposed solution for the ten-port calibration is based on the use of a multimode microwave oven as an impedance generator.

Figs. 6 and 7 show the online procedure diagram, which consists of sweeping the impedance space by displacing a high-loss dielectric sample inside the cavity over a nylon cartload along the main axis of the oven as described in [17]. Then, for each position of the cartload, both the $\left|S_{11}\right|$ measured with the VNA and the eight sampled voltage values corresponding to standing wave inside the ten-port are recorded at the computer. Fig. 7(b) shows the multimode oven and the PTFE carrying system dimensions.

Once the VNA and power detector measurements have been acquired, the neuron weights of the neural structure are obtained by an adaptive procedure, which is able to estimate the actual
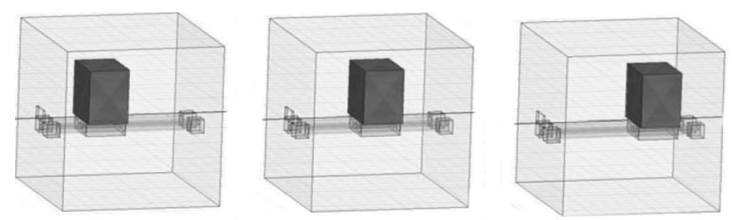

(a)

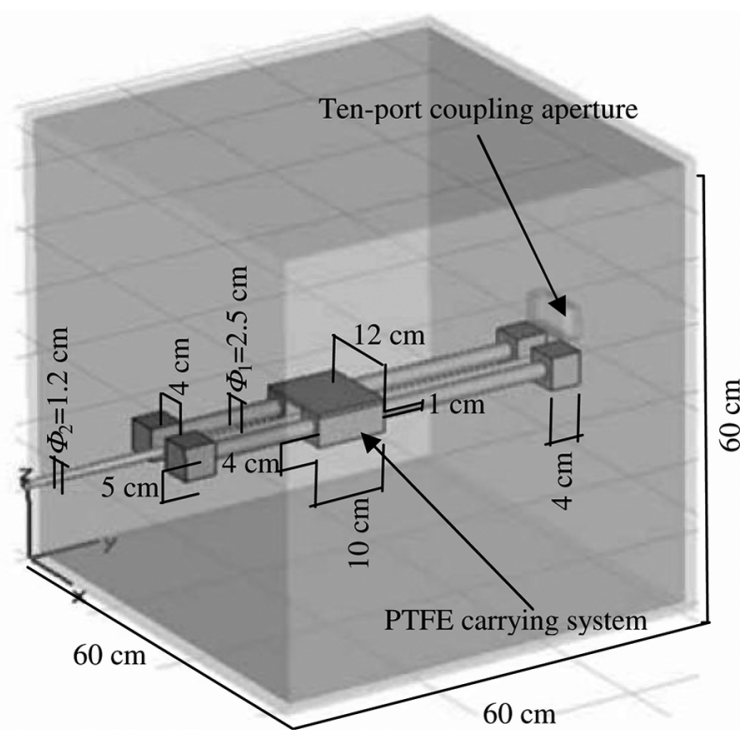

(b)

Fig. 7. (a) Impedance variation generated by displacements of a water sample inside the oven. (b) Multimode oven and PTFE carrying system dimensions.

values for magnitude and phase of $S_{11}$ from the power detectors' values. We refer in this case to the identification level.

Both the training and identification levels were implemented in MATLAB by using its Neural Network Toolbox.

\section{EXPERIMENTAL RESULTS}

The described calibration method, the waveguide reflectometer configuration, the electronic boards containing the power meters and the acquisition system were implemented and tested.

For the design of the network parameters, the number of both centroids and adaptive variances for each Gaussian function has matched the number of training steps or load iterations. The least mean square optimization algorithm has been implemented to find the weights that minimize the mean quadratic error during the training or calibration procedure. The number of faulty detectors has been varied in order to perform an analysis of the ten-port behavior under unusual conditions of the device.

\section{A. Training Procedure and Analysis of the Neural Structure}

The variable impedance generation needed for the training stage has been implemented in this study by using a $0.6 \mathrm{~m} \times$ $0.6 \mathrm{~m} \times 0.6 \mathrm{~m}$ cubic microwave oven with a movable dielectric load, as shown in Fig. 7(a), since sample position modification is capable of providing high impedances variations [17]. In this case, the dielectric load has consisted of a $1.5 \mathrm{~L}$ sample, which has provided $\left|S_{11}\right|$ values ranging from 0.1 to 0.8 . Five mm steps have been used for the position of the water load and, in this case, the sample has moved all along the axis shown in 


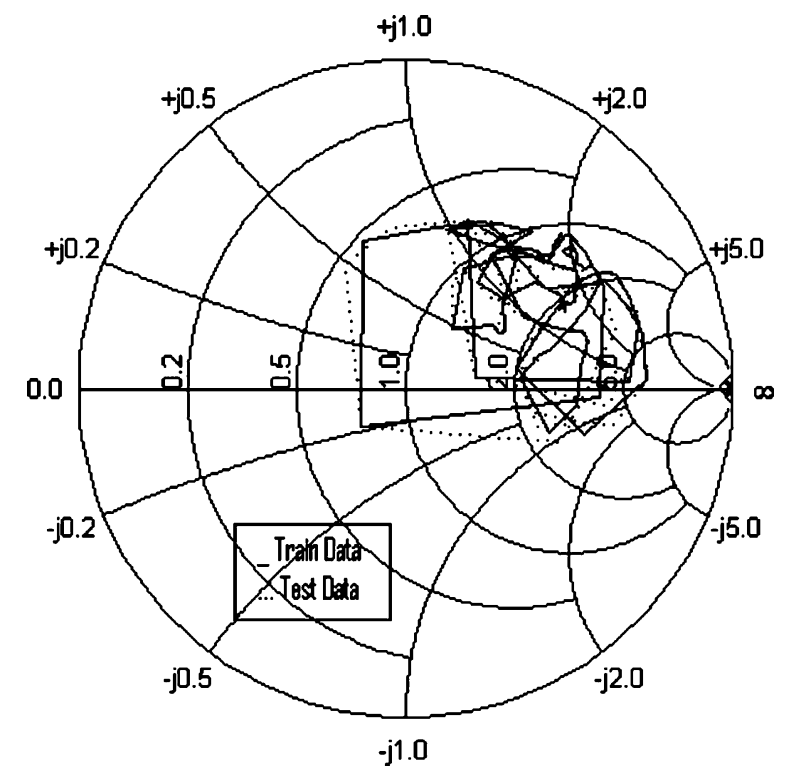

Fig. 8. Train and test data used for Figs. 10 and 11.

Fig. 7(a) by starting from the nearest point to the microwave source. This procedure has provided enough points to train the neural structure and to establish the relationship between the VNA and ten-port measurements.

Once the weights of the neural network have been calculated, the ten-port is calibrated and can autonomously measure magnitude and phase of $S_{11}$. Consequently, in the measurement stage, the network analyzer is no longer necessary although it has been used here for validation purposes.

\section{B. Estimation of $S_{11}$ by the Ten-Port Reflectometer}

Once the training data were acquired, the neural weights were learned by the neural network by using 100 neurons and 100 different values of $\left|S_{1} 1\right|$ from the VNA. Fig. 8 shows the comparison between the values of $S_{11}$ used to train the ten-port and the ones employed to test the sensor behavior.

Figs. 9 and 10 show the behavior, in module and phase, of the ten-port reflectometer versus VNA performance when values for $S_{11}$ that were not considered during the training process are used. The correspondence between both data is very high and therefore, the figures roughly follow a straight line with slope 1. Mean squared error was found to be in this case around $5.2 \times 10-5$. This indicates that the training procedure was successfully performed.

In order to evaluate the behavior of the ten-port when some detectors are operating in an inaccurate way, several tests have been carried out by using only a reduced number of diodes as valid sensors. The output of the faulty detectors is defined as a constant value for all the training positions. In the case of using just two valid detectors, the considered valid sensors were that placed at ports 5 and 8 in Fig. 2. When using only four detectors, the valid ones were that located at ports $3,5,7$, and 9 . The results are represented in Fig. 11, where 175 training steps and 59 validation measurements have been considered.

From curves in Fig. 11, one can observe that the proposed neural ten-port structure is able to predict the $S_{11}$ function with only four detectors, obtaining a low error level in the process.

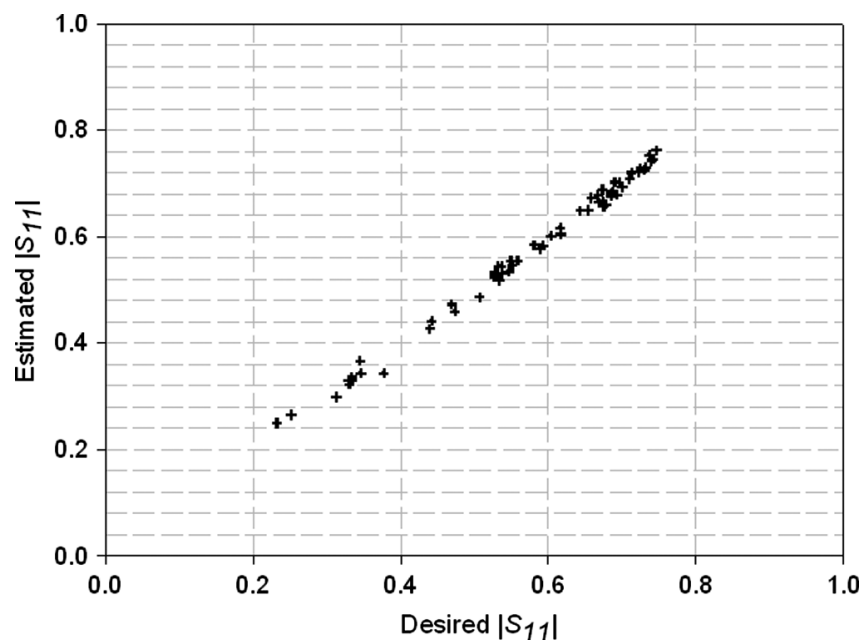

Fig. 9. $S_{11}$ module identification. Neural network response for testing data not used during the training stage.

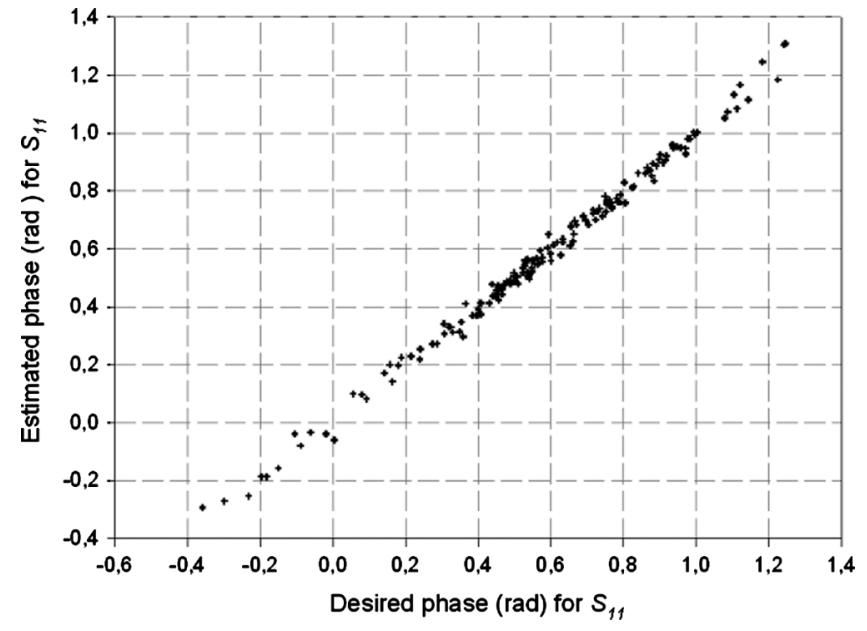

Fig. 10. $S_{11}$ phase identification. Neural network response for testing data not used during the training stage.

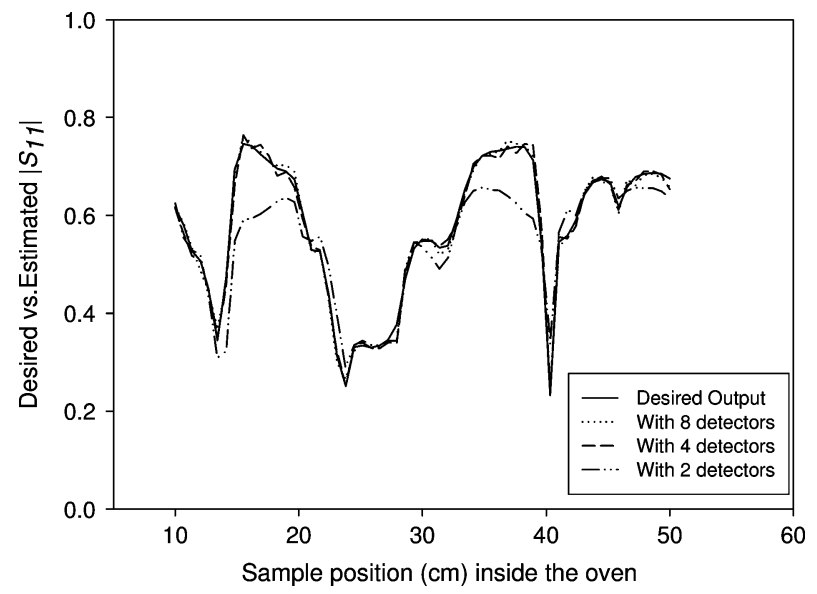

Fig. 11. Robustness testing. Behavior of the ten-port when some detectors are faulty.

As it has been demonstrated, this is one of the advantages of the ten-port, which is able to adapt its behavior when some detectors are broken. It can also be appreciated that a minimum of four 


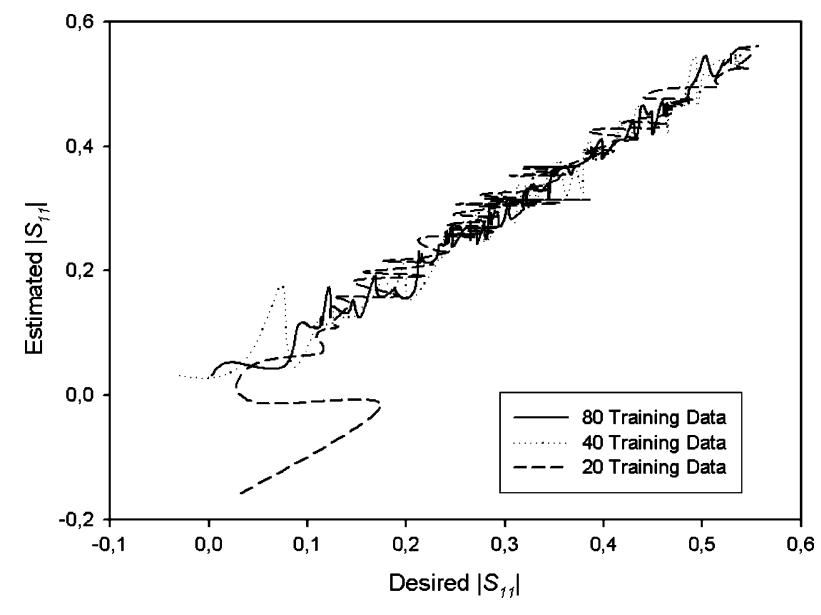

Fig. 12. Behavior of the ten-port as a function of the number of $S_{11}$ training data.

detectors is needed for a proper behavior since the result for two detectors is not acceptable.

A study of the required number of training data for a proper estimation of $S_{11}$ has been carried out. Fig. 12 shows the different behavior of the ten-port employed in this study as a function of the number of training data. In all cases, the number of neurons that has been employed is equal to the number of training $S_{11}$ values. However, in all cases, the number of test points was equal to 100 . One can conclude from Fig. 12 that the bigger the number of training data, the better the estimation of the reflection coefficient.

\section{CONCLUSIONS}

This study is focused on the design of a microwave reflectometer based on a waveguide ten-port structure and a calibration method that uses radial basis functions neural networks. To do this, a rectangular waveguide that contains eight power detectors for transforming the electromagnetic energy of the propagated $\mathrm{TE}_{10}$ mode into dc signals, a digital acquisition system and one neural network process have been implemented and tested.

The experimental trials have been carried out at low power and at $2.45 \mathrm{GHz}$. However, this method can be readily extended when high power is considered, by properly diminishing the coupling of the coaxial ports to the waveguide. Additionally, some other aspects should be considered such as frequency and power pulling when using magnetron as the microwave source. In this way, the device should identify the working frequency and therefore the neural architecture should be redefined.

The calibration has been performed at a fixed power and frequency and therefore the developed device can only work for these fixed values. However, the procedure can be extended for different values of frequency and power level since the network is capable of online adapting to new situations provided that they are properly trained. To do this, only a training process must be carried out for the specified frequency.

The performance of this device for a range of frequencies is proposed as a future work. It is important to remark that the different characteristics of the power detectors, the transmission lines and the manufactured board, introduce errors in the theoretical model of a reflectometer. However, in the proposed robust device, this fact is solved by the over-sampling of the standing wave inside the guide as well as the calibration procedure based on neural-network learning methods. In fact, it has been shown that the device is capable of adjusting its behavior when a power meter is not considered due to failure or saturation.

As a drawback, it must be pointed out that the training procedure requires a high number of load impedances to achieve a good performance of the neural network. However, this problem can be avoided by using automated procedures similar to the one employed in this study.

Finally, new studies are envisaged in order to extend the device performance for wider frequency bands and higher power levels.

\section{REFERENCES}

[1] A. C. Metaxas and R. J. Meredith, Industrial Microwave Heating. London, U.K.: Peregrinus, 1983.

[2] G. Colef, P. R. Karmel, and M. Ettenberg, "New in-situ calibration of diode detectors used in six-port network analyzers," IEEE Trans. Instrum. Meas., vol. 39, no. 1, pp. 201-204, Feb. 1990.

[3] E. J. Griffin, "Six-port reflectometer circuit comprising three directional couplers," Electron. Lett., vol. 18, no. 12, pp. 491-493, Jun. 1982.

[4] C. A. Hoer, "The six-port coupler: A new approach to measuring voltage, current, power impedance and phase," IEEE Trans. Instrum. Meas., vol. IM-21, no. 4, pp. 466-470, Nov. 1972.

[5] G. F. Engen, "The six-port reflectometer: An alternative network analyzer," IEEE Trans. Microw. Theory Tech., vol. MTT-25, no. 12, pp. 1075-1080, Dec. 1977.

[6] G. F. Engen, "A least squares solution for use in the six-port measurement technique," IEEE Trans. Microw. Theory Tech., vol. MTT-28, no. 12, pp. 1473-1477, Dec. 1980.

[7] G. F. Engen, "Calibrating the six-port reflectometer by means of sliding terminations," IEEE Trans. Microw. Theory Tech., vol. MTT-26, no. 12, pp. 951-957, Dec. 1978.

[8] F. Wiedmann, B. H. E. Bergeault, and L. Jallet, "A new robust method for six-port reflectometer calibration," IEEE Trans. Microw. Theory Tech., vol. 48, no. 10, pp. 927-931, Oct. 1999.

[9] J. Hesselbarth, F. Wiedmann, and B. Huyart, "Two new six-port reflectometers covering very large bandwidths," IEEE Trans. Microw. Theory Tech., vol. 46, no. 8, pp. 966-969, Aug. 1997.

[10] C. A. Hoer, "Performance of a dual six-port network analyzer," IEEE Trans. Microw. Theory Tech., vol. MTT-27, no. 9, pp. 993-998, Sep. 1979.

[11] S. R. Judah and W. Holmes, "A novel six-port calibration incorporating diode detector nonlinearity," in IEEE Instrum. Meas. Technol. Conf., May 1998, vol. 1, pp. 592-595.

[12] J. Moreau, A. El Idrissi, and C. Tibaudo, "Permittivity measurements of materials during heating by microwaves," Meas. Sci. Technol., vol. 5, pp. 996-1001, Aug. 1994.

[13] F. R. de Sousa, B. Huyart, and R. N. De Lima, "A new method for automatic calibration of 5-port reflectometers," J. Microw. Optoelectron., vol. 3, pp. 135-144, Oct. 2004.

[14] E. Eves, P. Kopyt, and V. V. Yakovlev, "Determination of complex permittivity with neural networks and FDTD modeling," Microw. Opt. Technol. Lett., vol. 40, no. 3, pp. 183-188, Feb. 2004.

[15] J. L. Pedreño-Molina, M. Pinzolas, and J. Monzó-Cabrera, "A new methodology for in-situ calibration of a neural network-based software sensor for $S$-parameter prediction in six-port reflectometers," Neurocomputing, vol. 69, pp. 2451-2455, Oct. 2006.

[16] D. Broomhead and D. Lowe, "Multivariable functional interpolation and adaptive networks," Complex Syst., vol. 2, pp. 321-355, 1988.

[17] M. E. Requena-Pérez, J. L. Pedreño-Molina, J. Monzó-Cabrera, and A. Díaz-Morcillo, "Multimode cavity efficiency optimization by optimum load location: Experimental approach," IEEE Trans. Microw. Theory Tech., vol. 53, no. 6, pp. 2838-2845, Jun. 2005. 


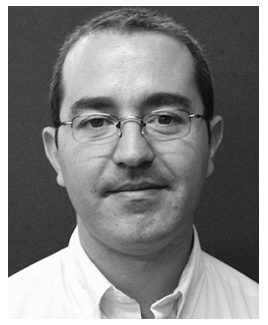

Juan Monzó-Cabrera was born in Elda (Alicante), Spain, on January 1973. He received the Dipl. Ing. and Ph.D. degrees in telecommunications engineering from the Universidad Politécnica de Valencia, Valencia, Spain, in 1998 and 2002, respectively.

From 1999 to 2000, he was a Research Assistant with the Microwave Heating Group (GCM). In 1999, he joined the Departamento de Tecnologías de la Información y las Comunicaciones, Universidad Politécnica de Cartagena, where he is currently an Associate Lecturer. He has coauthored over 50 papers in referred journals and conference proceedings. He holds several patents regarding microwave heating industrial processes. He is a reviewer of several international journals. His current research areas cover microwave-assisted heating and drying processes, microwave applicator design, and optimization and numerical techniques in electromagnetism.

Dr. Monzó-Cabrera is a Director of the Association of Microwave Power in Europe for Research and Education (AMPERE), a European-based organization devoted to the promotion of RF and microwave energy.

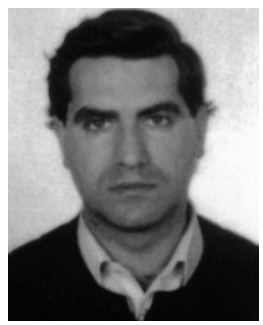

Juan L. Pedreño-Molina was born in Cartagena (Murcia), Spain, in 1969. He received the BASc. degree and $\mathrm{Ph} . \mathrm{D}$. in neurotechnology, control, and robotics from the Technical University of Madrid (UPM), Madrid, Spain, in 1984 and 2000, respectively.

He is an Assistant Professor of telecommunication engineering with the Technical University of Cartagena (UPCT), Cartagena, Spain. Since 1999, he has been with the Department of Information Technologies and Communications, UPCT. His research inter- ests are signal processing applied to optimization and control of nonlinear systems and modeling based on neural networks with applications to robotics and drying processes.

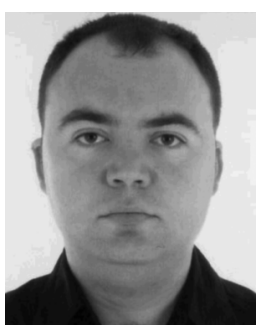

Antonio Lozano-Guerrero was born in El Verger, Spain. He received the Dipl. Ing. degree in telecommunications engineering from the Universidad Politécnica de Valencia (UPV), Valencia, Spain, and is currently working toward the Ph.D. degree at the Universidad Politécnica de Cartagena (UPCT), Cartagena, Spain.

From 2003 to 2004, he was a Research Assistant with the Department of Communications, UPV. In 2004, he joined the Department of Information Technologies and Communications, UPCT, where he is currently an Associate Lecturer. His current research areas are electromagnetic compatibility and numerical techniques in electromagnetism.

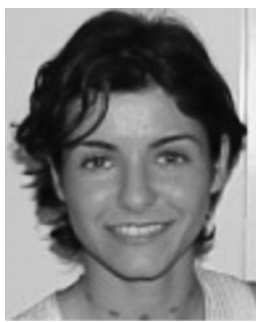

Ana Toledo-Moreo is an Assistant Lecturer with the Electronics Technology Department, Technical University of Cartagena, Cartagena, Spain. She has authored or coauthored numerous publications in international journals and conferences. Her research has been mainly focused on the development of low-level image processing algorithms for field-programmable gate arrays (FPGAs). She is also interested in artificial neural networks, principally in the improvement of learning algorithms. 